\title{
State Estimation for Fuzzy Sound Environment System with Finite Amplitude Fluctuation*
}

\author{
Akira Ikuta, Hisako Orimoto, Gerard Gallagher \\ Department of Management Information Systems, Prefectural University of Hiroshima, Hiroshima, Japan \\ Email: ikuta@pu-hiroshima.ac.jp
}

How to cite this paper: Ikuta, A., Orimoto, H. and Gallagher, G. (2017) State Estimation for Fuzzy Sound Environment System with Finite Amplitude Fluctuation. Journal of Software Engineering and Applications, 10, 625-638.

https://doi.org/10.4236/jsea.2017.107034

Received: April 19, 2017

Accepted: June 20, 2017

Published: June 23, 2017

Copyright $\odot 2017$ by authors and Scientific Research Publishing Inc. This work is licensed under the Creative Commons Attribution International License (CC BY 4.0).

http://creativecommons.org/licenses/by/4.0/

\section{Open Access}

\begin{abstract}
Internal physical mechanism of actual sound environment system is often difficult to recognize analytically, and it contains unknown structural characteristics. Furthermore, the observation data often contain fuzziness due to several causes and exhibit level saturation owing to the existence of a finite dynamic range. Therefore, it is necessary to propose a new state estimation method by considering fuzziness and finite amplitude fluctuation of observation data. In this paper, a method for estimating the specific signal for sound environment system with unknown structure is proposed in an appropriate form for the finite level range of the measured fuzzy observation data by introducing an expansion expression of probability distribution with Bata distribution in the first term and new type of membership function. The effectiveness of the proposed theoretical method is confirmed by applying it to the actual problem in the sound environment.
\end{abstract}

\section{Keywords}

Fuzzy Observation, Sound Environment System,

Finite Fluctuation Range, State Estimation

\section{Introduction}

The internal physical mechanism of actual sound environment system having a complicated relation to various factors is often difficult to recognize analytically, and it contains unknown structure. Furthermore, the stochastic process observed in the actual phenomenon exhibits complex fluctuation pattern and there are potentially various nonlinear correlations in addition to the linear correlation between input and output time series [1].

Furthermore, it is necessary to pay our attention on the fact that the observation data in the sound environment system often contain fuzziness due to several causes, for example, the permissible error of the accuracy in measurements, the ${ }^{*}$ State estimation for fuzzy sound environment system with finite amplitude fluctuation. 
quantized error in the digitization of observation data, and the existence of confidence limitation in measuring instruments. In our previous studies, state estimation methods for sound environment system based on fuzzy observation have been proposed by considering the standard Gaussian type membership function [2] [3]. On the other hand, owing to a finite range of the amplitude fluctuation and/or a definite dynamic range of measurement instrument, only the information defined within a finite range usually can be obtained as reliable data in the measurement of real sound environment [4] [5]. In this situation, in order to evaluate more precisely the objective sound environment system, it is necessary to introduce a fuzzy theory to estimate the waveform fluctuation of the specific signal based on the observed data with fuzziness and amplitude saturation.

As a typical method in the state estimation problem, the Kalman filtering theory and its extended filter are well known [6] [7] [8] [9] [10]. Furthermore, several state estimation methods for stochastic system with non-Gaussian fluctuations have previously been proposed [11] [12] [13] [14]. These theories are originally based on the additive model of the specific signal and an external noise. The actual sound environment systems often contain unknown characteristics in the relationship between the state variable and the observation. Furthermore, the observation data often contain fuzziness and amplitude saturation.

In this study, based on the fuzzy observations, a Bayesian filter for estimating the specific signal of sound environment systems with unknown structural characteristic is theoretically proposed in an appropriate form for the finite amplitude range of the measured data. More specifically, complex sound environment systems which have to be treated as the systems with unknown characteristics are paid our attention. By introducing the orthogonal expansion expression of the probability distribution with a Beta distribution as the first term, and a new type of membership function, which are suitable for the finite amplitude fluctuation range of the signal and observation, a method to estimate the waveform fluctuation of the specific signal based on the fuzzy observation data is proposed. After adopting a previously reported expansion expression of the conditional probability distribution as the correlation information between the specific signal and observation [1], by regarding the expansion coefficients as unknown parameters and introducing probability measure of fuzzy events [15], these parameters are estimated simultaneously with the specific signal based on the fuzzy observation.

Finally, the effectiveness of the proposed Bayesian filter focusing on the relationship between variables is confirmed experimentally too by applying the theory to the estimation of sound level based on the observation data containing fuzziness and amplitude saturation.

\section{Theoretical Consideration}

\subsection{Formulation of Sound Environment System with Fuzzy Observation}

Let $x_{k}$ and $y_{k}$ be the input and output signals at a discrete time $k$ for a sound 
environment system. It is assumed that there are complex nonlinear relationships between $x_{k}$ and $y_{k}$, which are difficult to find a fundamental relationship between them. Since the system characteristics are unknown, a system model in the form of a conditional probability is adopted. More precisely, attention is focused on the joint probability distribution function $P\left(x_{k}, y_{k}\right)$ reflecting all linear and non-linear correlation information between $x_{k}$ and $y_{k}$. Expanding the joint probability distribution function $P\left(x_{k}, y_{k}\right)$ in an orthogonal form based on the product of $P\left(x_{k}\right)$ and $P\left(y_{k}\right)$, the following expression can be derived.

$$
P\left(x_{k}, y_{k}\right)=P\left(x_{k}\right) P\left(y_{k}\right) \sum_{r=0}^{\infty} \sum_{s=0}^{\infty} A_{r s} \theta_{r}^{(1)}\left(x_{k}\right) \theta_{s}^{(2)}\left(y_{k}\right)
$$

with

$$
A_{r s} \equiv\left\langle\theta_{r}^{(1)}\left(x_{k}\right) \theta_{s}^{(2)}\left(y_{k}\right)\right\rangle
$$

where \langle\rangle denotes the averaging operation on the variables. The linear and non-linear correlation information between $x_{k}$ and $y_{k}$ is reflected hierarchically in each expansion coefficient $A_{r s}$. The functions $\theta_{r}^{(1)}\left(x_{k}\right)$ and $\theta_{s}^{(2)}\left(y_{k}\right)$ are orthonormal polynomials with the weighting functions $P\left(x_{k}\right)$ and $P\left(y_{k}\right)$ respectively, and satisfy the following orthonormal conditions:

$$
\begin{aligned}
& \int \theta_{r}^{(1)}\left(x_{k}\right) \theta_{r^{\prime}}^{(1)}\left(x_{k}\right) P\left(x_{k}\right) \mathrm{d} x_{k}=\delta_{r r^{\prime}} \\
& \int \theta_{s}^{(2)}\left(y_{k}\right) \theta_{s^{\prime}}^{(2)}\left(y_{k}\right) P\left(y_{k}\right) \mathrm{d} y_{k}=\delta_{s s^{\prime}}
\end{aligned}
$$

These orthonormal polynomials can be decomposed by using Schmidt's orthogonalization [16]. From Equation (1), the conditional probability distribution function $P\left(y_{k} \mid x_{k}\right)$ is given as

$$
P\left(y_{k} \mid x_{k}\right)=\frac{P\left(x_{k}, y_{k}\right)}{P\left(x_{k}\right)}=P\left(y_{k}\right) \sum_{r=0}^{R} \sum_{s=0}^{S} A_{r s} \theta_{r}^{(1)}\left(x_{k}\right) \theta_{s}^{(2)}\left(y_{k}\right)
$$

Though Equation (5) is originally infinite series expansion, finite expansion series with $r \leq R$ and $s \leq S$ is adopted because only finite expansion coefficients are available and the consideration of the expansion coefficients from the first few terms is usually sufficient in practice. The expansion coefficients defined by Equation (2) satisfy the following conditions:

$$
A_{00}=1, A_{r 0}=A_{0 s}=0(r, s=1,2, \cdots)
$$

Furthermore, let $z_{k}$ be the fuzzy observation obtained from the output $y_{k}$ with a finite fluctuation range $[a, b]$ owing to a definite dynamic range of measurement instrument. The fuzziness of $z_{k}$ is characterized by a membership function $\mu_{z_{k}}\left(y_{k}\right)$.

Since the objective system contains an unknown specific signal and unknown structure, the expansion coefficients $A_{r s}$ expressing hierarchically the correlation relationship between $x_{k}$ and $y_{k}$ must be estimated on the basis of the fuzzy observation $z_{k}$. Considering the expansion coefficients $A_{r s}$ as unknown parameter vector $\mathbf{a}$ : 


$$
\begin{gathered}
\mathbf{a} \equiv\left(a_{1}, a_{2}, \cdots, a_{I}\right) \equiv\left(\mathbf{a}_{(1)}, \mathbf{a}_{(2)}, \cdots, \mathbf{a}_{(S)}\right) \\
\mathbf{a}_{(s)} \equiv\left(A_{1 s}, A_{2 s}, \cdots, A_{R s}\right),(s=1,2, \cdots, S)
\end{gathered}
$$

the following simple dynamical models are introduced for the simultaneous estimation of the parameters with the specific signal $x_{k}$ :

$$
\begin{gathered}
\mathbf{a}_{k+1}=\mathbf{a}_{k} \\
\left(\mathbf{a}_{k} \equiv\left(a_{1, k}, a_{2, k}, \cdots, a_{I, k}\right) \equiv\left(\mathbf{a}_{(1), k}, \mathbf{a}_{(2), k}, \cdots, \mathbf{a}_{(S), k}\right)\right)
\end{gathered}
$$

where $I(=R \cdot S)$ is the number of unknown expansion coefficients to be estimated.

On the other hand, the following time transition model for the input signal is generally established.

$$
x_{k+1}=F x_{k}+G u_{k}
$$

where $u_{k}$ is the random input with mean 0 and variance $\sigma_{u}^{2}$. Two parameters $F$ and $G$ are estimated by using an auto-correlation technique [16].

A method to estimate $x_{k}$ adaptively based on the fuzzy observation $z_{k}$ is derived in this study by introducing probability measure of fuzzy events [15] and the expansion series expressions of the conditional probability distribution function in Equation (5).

\subsection{Derivation of Estimation Algorithm Based on Fuzzy Observation with Amplitude Saturation}

In order to derive an estimation algorithm for a specific signal $x_{k}$, based on the successive observations of fuzzy data $z_{k}$, we focus our attention on Bayes' theorem for the conditional probability distribution [12]. Since the parameter $\mathbf{a}_{k}$ is also unknown, the conditional probability distribution of $x_{k}$ and $\mathbf{a}_{k}$ is considered.

$$
P\left(x_{k}, \mathbf{a}_{k} \mid Z_{k}\right)=\frac{P\left(x_{k}, \mathbf{a}_{k}, z_{k} \mid Z_{k-1}\right)}{P\left(z_{k} \mid Z_{k-1}\right)}
$$

where $Z_{k}\left(=\left(z_{1}, z_{2}, \cdots, z_{k}\right)\right)$ is a set of fuzzy observation data up to a time $k$. After applying probability measure of fuzzy events [15] to the right side of Equation (10), expanding it in a general form of the statistical orthogonal expansion series [12], the conditional probability density function $P\left(x_{k}, \mathbf{a}_{k} \mid Z_{k}\right)$ can be expressed as:

$$
\begin{aligned}
& P\left(x_{k}, \mathbf{a}_{k} \mid Z_{k}\right)=\frac{\int_{a}^{b} \mu_{z_{k}}\left(y_{k}\right) P\left(x_{k}, \mathbf{a}_{k}, y_{k} \mid Z_{k-1}\right) \mathrm{d} y_{k}}{\int_{a}^{b} \mu_{z_{k}}\left(y_{k}\right) P\left(y_{k} \mid Z_{k-1}\right) \mathrm{d} y_{k}} \\
& =\frac{\sum_{l=0}^{\infty} \sum_{\mathbf{m}=\mathbf{0}}^{\infty} \sum_{n=0}^{\infty} B_{l \mathbf{m} n} P_{0}\left(x_{k} \mid Z_{k-1}\right) P_{0}\left(\mathbf{a}_{k} \mid Z_{k-1}\right) \varphi_{l}^{(1)}\left(x_{k}\right) \varphi_{\mathbf{m}}^{(2)}\left(\mathbf{a}_{k}\right) I_{n}\left(z_{k}\right)}{\sum_{n=0}^{\infty} B_{00 n} I_{n}\left(z_{k}\right)}
\end{aligned}
$$

with

$$
I_{n}\left(z_{k}\right) \equiv \int_{a}^{b} \mu_{z_{k}}\left(y_{k}\right) P_{0}\left(y_{k} \mid Z_{k-1}\right) \varphi_{n}^{(3)}\left(y_{k}\right) \mathrm{d} y_{k}
$$




$$
\begin{gathered}
B_{l \mathbf{m} n} \equiv\left\langle\varphi_{l}^{(1)}\left(x_{k}\right) \varphi_{\mathbf{m}}^{(2)}\left(\mathbf{a}_{k}\right) \varphi_{n}^{(3)}\left(y_{k}\right) \mid Z_{k-1}\right\rangle \\
\left(\mathbf{m} \equiv\left(m_{1}, m_{2}, \cdots, m_{I}\right)\right) .
\end{gathered}
$$

The functions $\varphi_{l}^{(1)}\left(x_{k}\right), \varphi_{\mathbf{m}}^{(2)}\left(\mathbf{a}_{k}\right)$ and $\varphi_{n}^{(3)}\left(y_{k}\right)$ are the orthogonal polynomials of degrees $l, \mathbf{m}$ and $n$ with weighting functions $P_{0}\left(x_{k} \mid Z_{k-1}\right)$, $P_{0}\left(\mathbf{a}_{k} \mid Z_{k-1}\right)$ and $P_{0}\left(y_{k} \mid Z_{k-1}\right)$, which can be artificially chosen as the probability density functions describing the dominant parts of $P\left(x_{k} \mid Z_{k-1}\right)$, $P\left(\mathbf{a}_{k} \mid Z_{k-1}\right)$ and $P\left(y_{k} \mid Z_{k-1}\right)$. These three functions must satisfy the following orthonormal relationships:

$$
\begin{gathered}
\int_{-\infty}^{\infty} \varphi_{l}^{(1)}\left(x_{k}\right) \varphi_{l^{\prime}}^{(1)}\left(x_{k}\right) P_{0}\left(x_{k} \mid Z_{k-1}\right) \mathrm{d} x_{k}=\delta_{l l^{\prime}} \\
\int_{-\infty}^{\infty} \varphi_{\mathbf{m}}^{(2)}\left(\mathbf{a}_{k}\right) \varphi_{\mathbf{m}^{\prime}}^{(2)}\left(\mathbf{a}_{k}\right) P_{0}\left(\mathbf{a}_{k} \mid Z_{k-1}\right) \mathrm{d} \mathbf{a}_{k}=\prod_{i=1}^{I} \delta_{m_{i} m_{i}^{\prime}} \\
\int_{a}^{b} \varphi_{n}^{(3)}\left(y_{k}\right) \varphi_{n^{\prime}}^{(3)}\left(y_{k}\right) P_{0}\left(y_{k} \mid Z_{k-1}\right) \mathrm{d} y_{k}=\delta_{n n^{\prime}}
\end{gathered}
$$

Based on Equation (11), the recurrence algorithm for estimating an arbitrary $(L, \mathbf{M})$ th order polynomial type function $f_{L, \mathbf{M}}\left(x_{k}, \mathbf{a}_{k}\right)$ of $x_{k}$ and $\mathbf{a}_{k}$ can be derived as follows:

$$
\hat{f}_{L, \mathbf{M}}\left(x_{k}, \mathbf{a}_{k}\right) \equiv\left\langle f_{L, \mathbf{M}}\left(x_{k}, \mathbf{a}_{k}\right) \mid Z_{k}\right\rangle=\frac{\sum_{l=0}^{L} \sum_{\mathbf{m}=0}^{\mathbf{M}} \sum_{n=0}^{\infty} C_{l \mathbf{m}}^{L \mathbf{M}} B_{l \mathbf{m} n} I_{n}\left(z_{k}\right)}{\sum_{n=0}^{\infty} B_{00 n} I_{n}\left(z_{k}\right)}
$$

where $C_{l \mathbf{m}}^{L \mathbf{M}}$ is the expansion coefficient determined by the equality:

$$
f_{L, \mathbf{M}}\left(x_{k}, \mathbf{a}_{k}\right)=\sum_{l=0}^{L} \sum_{\mathbf{m}=\mathbf{0}}^{\mathbf{M}} C_{l \mathbf{m}}^{L \mathbf{M}} \varphi_{l}^{(1)}\left(x_{k}\right) \varphi_{\mathbf{m}}^{(2)}\left(\mathbf{a}_{k}\right)
$$

In order to make the general theory for estimation algorithm more concrete, the well-known Gaussian distribution is adopted as $P_{0}\left(x_{k} \mid Z_{k-1}\right)$ and $P_{0}\left(\mathbf{a}_{k} \mid Z_{k-1}\right)$, because this probability density function is the most standard one.

$$
\begin{gathered}
P_{0}\left(x_{k} \mid Z_{k-1}\right)=N\left(x_{k} ; x_{k}^{*}, \Gamma_{x_{k}}\right) \\
P_{0}\left(\mathbf{a}_{k} \mid Z_{k-1}\right)=\prod_{i=1}^{I} N\left(a_{i, k} ; a_{i, k}^{*}, \Gamma_{a_{i, k}}\right)
\end{gathered}
$$

with

$$
\begin{gathered}
N\left(x ; \mu, \sigma^{2}\right) \equiv \frac{1}{\sqrt{2 \pi \sigma^{2}}} \exp \left\{-\frac{(x-\mu)^{2}}{2 \sigma^{2}}\right\} \\
x_{k}^{*} \equiv\left\langle x_{k} \mid Z_{k-1}\right\rangle, \quad \Gamma_{x_{k}} \equiv\left\langle\left(x_{k}-x_{k}^{*}\right)^{2} \mid Z_{k-1}\right\rangle \\
a_{i, k}^{*} \equiv\left\langle a_{i, k}^{*} \mid Z_{k-1}\right\rangle, \quad \Gamma_{a_{i, k}} \equiv\left\langle\left(a_{i, k}-a_{i, k}^{*}\right)^{2} \mid Z_{k-1}\right\rangle
\end{gathered}
$$

Furthermore, the Bata distribution [17] suitable for the random variable with a finite fluctuation range $[a, b]$ is adopted as $P_{0}\left(y_{k} \mid Z_{k-1}\right)$.

$$
P_{0}\left(y_{k} \mid Z_{k-1}\right)=\frac{1}{B\left(\gamma_{k}, \alpha_{k}-\gamma_{k}+1\right)(b-a)}\left(\frac{y_{k}-a}{b-a}\right)^{\gamma_{k}-1}\left(\frac{b-y_{k}}{b-a}\right)^{\alpha_{k}-\gamma_{k}}
$$


with

$$
\begin{gathered}
B(p, q) \equiv \int_{0}^{1} t^{p-1}(1-t)^{q-1} \mathrm{~d} t=\Gamma(p) \Gamma(q) / \Gamma(p+q) ; \text { Beta function } \\
\alpha_{k} \equiv \frac{\left(y_{k}^{*}-a\right)\left(b-y_{k}^{*}\right)}{\Omega_{k}}-2 \\
\gamma_{k} \equiv \frac{y_{k}^{*}-a}{b-a}\left\{\frac{\left(y_{k}^{*}-a\right)\left(b-y_{k}^{*}\right)}{\Omega_{k}}-1\right\} \\
y_{k}^{*} \equiv\left\langle y_{k} \mid Z_{k-1}\right\rangle, \Omega_{k} \equiv\left\langle\left(y_{k}-y_{k}^{*}\right)^{2} \mid Z_{k-1}\right\rangle
\end{gathered}
$$

where $\Gamma(\cdot)$ is the Gamma function. Then, the orthonormal functions with the weighting probability density functions in Equations (19) and (21) can be given by

$$
\begin{gathered}
\varphi_{l}^{(1)}\left(x_{k}\right)=\frac{1}{\sqrt{l !}} H_{l}\left(\frac{x_{k}-x_{k}^{*}}{\sqrt{\Gamma_{x_{k}}}}\right) \\
\varphi_{\mathbf{m}}^{(2)}\left(\mathbf{a}_{k}\right)=\prod_{i=1}^{I} \frac{1}{\sqrt{m_{i} !}} H_{m_{i}}\left(\frac{a_{i, k}-a_{i, k}^{*}}{\Gamma_{a_{i, k}}}\right) \\
\varphi_{n}^{(3)}\left(y_{k}\right)=\sqrt{\frac{\Gamma\left(\alpha_{k}-\gamma_{k}+1\right)\left(\alpha_{k}+2 n\right) \Gamma\left(\alpha_{k}+n\right) \Gamma\left(\gamma_{k}+n\right)}{\Gamma\left(\alpha_{k}+1\right) n ! \Gamma\left(n+\alpha_{k}-\gamma_{k}+1\right) \Gamma\left(\gamma_{k}\right)}} G_{n}\left(\alpha_{k}, \gamma_{k} ; \frac{y_{k}-a}{b-a}\right)
\end{gathered}
$$

where $H_{l}(\cdot)$ is the Hermite polynomial with th order [18] and $G_{n}(\cdot)$ is the Jacobi polynomial with $n$th order [4]. As the membership function, the following function matching to the Beta distribution is newly introduced.

$$
\begin{gathered}
\mu_{z_{k}}\left(y_{k}\right)=w_{k}^{-s}\left(1-w_{k}\right)^{s\left(w_{k}-1\right) / w_{k}}\left(\frac{y_{k}-a}{b-a}\right)^{s}\left(\frac{b-y_{k}}{b-a}\right)^{s\left(1-w_{k}\right) / w_{k}} \\
\left(w_{k} \equiv\left(z_{k}-a\right) /(b-a)\right)
\end{gathered}
$$

where $s$ is a parameter.

Accordingly, Equation (12) can be given by

$$
\begin{aligned}
& I_{n}\left(z_{k}\right)=w_{k}^{-s}\left(1-w_{k}\right)^{s\left(w_{k}-1\right) / w_{k}} \frac{\Gamma\left(\alpha_{k}+1\right)}{\Gamma\left(\gamma_{k}\right) \Gamma\left(\alpha_{k}-\gamma_{k}+1\right)} \frac{\Gamma\left(A_{k}\right) \Gamma\left(B_{k}-A_{k}+1\right)}{\Gamma\left(B_{k}+1\right)} \\
& \cdot \int_{a}^{b} \frac{\Gamma\left(B_{k}+1\right)}{\Gamma\left(A_{k}\right) \Gamma\left(B_{k}-A_{k}+1\right)}\left(\frac{y_{k}-a}{b-a}\right)^{A_{k}-1}\left(\frac{b-y_{k}}{b-a}\right)^{B_{k}-A_{k}} \\
& \cdot \sqrt{\frac{\Gamma\left(\alpha_{k}-\gamma_{k}+1\right)\left(\alpha_{k}+2 n\right) \Gamma\left(\alpha_{k}+n\right) \Gamma\left(\gamma_{k}+n\right)}{\Gamma\left(\alpha_{k}+1\right) n ! \Gamma\left(n+\alpha_{k}-\gamma_{k}+1\right) \Gamma\left(\gamma_{k}\right)}} \sum_{r=0}^{n} d_{n r} G_{r}\left(B_{k}, A_{k} ; \frac{y_{k}-a}{b-a}\right) d y_{k}
\end{aligned}
$$

with

$$
A_{k} \equiv \gamma_{k}+s, \quad B_{k} \equiv s\left(1-w_{k}\right) / w_{k}+\alpha_{k}+s
$$

The fuzzy data $z_{k}$ are reflected in $w_{k}$ and $B_{k}$. Furthermore, $d_{n r}$ $(r=0,1,2, \cdots, n)$ are the expansion coefficients in the equality on Jacobi polynomial:

$$
G_{n}\left(\alpha_{k}, \gamma_{k} ; \frac{y_{k}-a}{b-a}\right)=\sum_{r=0}^{n} d_{n r} G_{r}\left(B_{k}, A_{k} ; \frac{y_{k}-a}{b-a}\right)
$$


By considering the orthonormal condition of Jacobi polynomial [4], Equation (27) can be expressed as follows:

$$
\begin{aligned}
I_{n}\left(z_{k}\right)= & w_{k}^{-s}\left(1-w_{k}\right)^{s\left(w_{k}-1\right) / w_{k}} \frac{\Gamma\left(\alpha_{k}+1\right)}{\Gamma\left(\gamma_{k}\right) \Gamma\left(\alpha_{k}-\gamma_{k}+1\right)} \frac{\Gamma\left(A_{k}\right) \Gamma\left(B_{k}-A_{k}+1\right)}{\Gamma\left(B_{k}+1\right)} \\
& \cdot \sqrt{\frac{\Gamma\left(\alpha_{k}-\gamma_{k}+1\right)\left(\alpha_{k}+2 n\right) \Gamma\left(\alpha_{k}+n\right) \Gamma\left(\gamma_{k}+n\right)}{\Gamma\left(\alpha_{k}+1\right) n ! \Gamma\left(n+\alpha_{k}-\gamma_{k}+1\right) \Gamma\left(\gamma_{k}\right)}} d_{n 0},
\end{aligned}
$$

where a few concrete expressions of $d_{n 0}$ in Equation (30) can be expressed as follows:

$$
\begin{gathered}
d_{00}=1, \quad d_{10}=1-\frac{\alpha_{k}+1}{\gamma_{k}} \frac{A_{k}}{B_{k}+1} \\
d_{20}=1-2 \frac{\alpha_{k}+2}{\gamma_{k}} \frac{A_{k}}{B_{k}+1}+\frac{\left(\alpha_{k}+3\right)\left(\alpha_{k}+2\right)}{\left(\gamma_{k}+1\right) \gamma_{k}} \frac{\left(A_{k}+1\right) A_{k}}{\left(B_{k}+2\right)\left(B_{k}+1\right)} \\
d_{30}=1-3 \frac{\alpha_{k}+3}{\gamma_{k}} \frac{A_{k}}{B_{k}+1}+3 \frac{\left(\alpha_{k}+4\right)\left(\alpha_{k}+3\right)}{\left(\gamma_{k}+1\right) \gamma_{k}} \frac{\left(A_{k}+1\right) A_{k}}{\left(B_{k}+2\right)\left(B_{k}+1\right)} \\
-\frac{\left(\alpha_{k}+5\right)\left(\alpha_{k}+4\right)\left(\alpha_{k}+3\right)}{\left(\gamma_{k}+2\right)\left(\gamma_{k}+1\right) \gamma_{k}} \frac{\left(A_{k}+2\right)\left(A_{k}+1\right) A_{k}}{\left(B_{k}+3\right)\left(B_{k}+2\right)\left(B_{k}+1\right)} .
\end{gathered}
$$

In two special cases when $f_{1,0}\left(x_{k}, \mathbf{a}_{k}\right)=x_{k}, f_{2,0}\left(x_{k}, \mathbf{a}_{k}\right)=\left(x_{k}-\hat{x}_{k}\right)^{2}$, estimates related to mean and variance of the specific signal are expressed as follows:

$$
\begin{gathered}
\hat{x}_{k} \equiv\left\langle x_{k} \mid Z_{k}\right\rangle=\frac{\sum_{n=0}^{\infty}\left\{B_{00 n} C_{00}^{10}+B_{10 n} C_{10}^{10}\right\} I_{n}\left(z_{k}\right)}{\sum_{n=0}^{\infty} B_{00 n} I_{n}\left(z_{k}\right)} \\
P_{k} \equiv\left\langle\left(x_{k}-\hat{x}_{k}\right)^{2} \mid Z_{k}\right\rangle=\frac{\sum_{n=0}^{\infty}\left\{B_{00 n} C_{00}^{20}+B_{10 n} C_{10}^{20}+B_{20 n} C_{20}^{20}\right\} I_{n}\left(z_{k}\right)}{\sum_{n=0}^{\infty} B_{00 n} I_{n}\left(z_{k}\right)}
\end{gathered}
$$

with

$$
\begin{gathered}
C_{00}^{10}=x_{k}^{*}, C_{10}^{10}=\sqrt{\Gamma_{x_{k}}}, \\
C_{00}^{20}=\Gamma_{x_{k}}+\left(x_{k}^{*}-\hat{x}_{k}\right)^{2}, C_{10}^{20}=2 \sqrt{\Gamma_{x_{k}}}\left(x_{k}^{*}-\hat{x}_{k}\right), C_{20}^{20}=\sqrt{2} \Gamma_{x_{k}} .
\end{gathered}
$$

Using the property of conditional expectation and Equation (5), the two variables $y_{k}^{*}$ and $\Omega_{k}$ in Equation (22) can be expressed in functional forms on predictions of $x_{k}$ and $\mathbf{a}_{k}$ at a discrete time $k-1$ (i.e. the expectation value of arbitrary functions of $x_{k}$ and $\mathbf{a}_{k}$ conditioned by $\left.Z_{k-1}\right)$,as follows:

$$
\begin{aligned}
y_{k}^{*} & =\left\langle\left\langle y_{k} \mid x_{k}, Z_{k-1}\right\rangle \mid Z_{k-1}\right\rangle \\
& =\left\langle\int y_{k} P\left(y_{k} \mid x_{k}\right) \mathrm{d} y_{k} \mid Z_{k-1}\right\rangle \\
& =\left\langle\sum_{r=0}^{\infty} \sum_{s=0}^{1} e_{1 s} A_{r s} \theta_{r}^{(1)}\left(x_{k}\right) \mid Z_{k-1}\right\rangle \\
& =\sum_{s=0}^{1} e_{1 s}\left\langle\mathbf{A}_{(s), k} \Theta\left(x_{k}\right) \mid Z_{k-1}\right\rangle,
\end{aligned}
$$




$$
\begin{aligned}
\Omega_{k} & =\left\langle\int\left(y_{k}-y_{k}^{*}\right)^{2} P\left(y_{k} \mid x_{k}\right) \mathrm{d} y_{k} \mid Z_{k-1}\right\rangle \\
& =\left\langle\sum_{r=0}^{\infty} \sum_{s=0}^{2} e_{2 s} A_{r s} \theta_{r}^{(1)}\left(x_{k}\right) \mid Z_{k-1}\right\rangle \\
& =\sum_{s=0}^{2} e_{2 s}\left\langle\mathbf{A}_{(s), k} \Theta\left(x_{k}\right) \mid Z_{k-1}\right\rangle
\end{aligned}
$$

with

$$
\begin{gathered}
\mathbf{A}_{(s), k} \equiv\left(0, \mathbf{a}_{(s), k}\right),(s=1,2, \cdots), \quad \mathbf{A}_{(0), k} \equiv(1,0,0, \cdots, 0) \\
\Theta\left(x_{k}\right) \equiv\left(\theta_{0}^{(1)}\left(x_{k}\right), \theta_{1}^{(1)}\left(x_{k}\right), \cdots, \theta_{R}^{(1)}\left(x_{k}\right)\right)^{\mathrm{T}}
\end{gathered}
$$

where $\mathrm{T}$ denotes the transpose of a matrix. The coefficients $e_{1 s}$ and $e_{2 s}$ in Equations (35) and (36) are determined in advance by expanding $y_{k}$ and $\left(y_{k}-y_{k}^{*}\right)^{2}$ in the following orthogonal series forms:

$$
y_{k}=\sum_{i=0}^{1} e_{1 i} \theta_{i}^{(2)}\left(y_{k}\right),\left(y_{k}-y_{k}^{*}\right)^{2}=\sum_{i=0}^{2} e_{2 i} \theta_{i}^{(2)}\left(y_{k}\right)
$$

Furthermore, using Equation (5) and the orthonormal condition of Equation (4), each expansion coefficient $B_{l m n}$ defined by Equation (13) can be obtained through the similar calculation process to Equations (35) and (36), as follows:

$$
\begin{aligned}
& B_{l \mathbf{m} n}=\left\langle\varphi_{l}^{(1)}\left(x_{k}\right) \varphi_{\mathbf{m}}^{(2)}\left(\mathbf{a}_{k}\right) \int \varphi_{n}^{(3)}\left(y_{k}\right) P\left(y_{k} \mid x_{k}\right) \mathrm{d} y_{k} \mid Z_{k-1}\right\rangle \\
&=\left\langle\varphi_{l}^{(1)}\left(x_{k}\right) \varphi_{\mathbf{m}}^{(2)}\left(\mathbf{a}_{k}\right) \sum_{r=0}^{\infty} \sum_{s=0}^{n} e_{n s} A_{r s} \theta_{r}^{(1)}\left(x_{k}\right) \mid Z_{k-1}\right\rangle \\
&=\sum_{s=0}^{n} e_{n s}\left\langle\varphi_{l}^{(1)}\left(x_{k}\right) \varphi_{\mathbf{m}}^{(2)}\left(\mathbf{a}_{k}\right) \mathbf{A}_{(s), k} \Theta\left(x_{k}\right) \mid Z_{k-1}\right\rangle \\
&\left(\varphi_{n}^{(3)}\left(y_{k}\right)=\sum_{i=0}^{n} e_{n i} \theta_{i}^{(2)}\left(y_{k}\right), \quad e_{n i}: \text { appropriate coefficients }\right) .
\end{aligned}
$$

In the above, the expansion coefficient $B_{l \mathrm{~m} n}$ can be given by the predictions of $x_{k}$ and $\mathbf{a}_{k}$.

Finally, by considering Equation (9), the prediction step which is essential to perform the recurrence estimation can be given by

$$
\begin{gathered}
x_{k+1}^{*} \equiv\left\langle x_{k+1} \mid Z_{k}\right\rangle=F \hat{x}_{k}+G\left\langle u_{k}\right\rangle \\
\Gamma_{k+1} \equiv\left\langle\left(x_{k+1}-x_{k+1}^{*}\right)^{2} \mid Z_{k}\right\rangle=F^{2} P_{k}+G^{2}\left\langle\left(u_{k}-\left\langle u_{k}\right\rangle\right)^{2}\right\rangle
\end{gathered}
$$

By replacing $k$ with $k+1$, the recurrence estimation of $x_{k}$ can be achieved.

\section{Experimental Consideration}

In order to confirm the effectiveness of the proposed method, it was applied to real data observed in a sound environment system. Acoustic signals observed by two microphones in indoors and outdoors for a house were adopted as input and output data for the sound insulation system shown by the frame in Figure 1. The rock music was selected as an input signal by considering the aggravation of "Karaoke" noise pollution problem. 


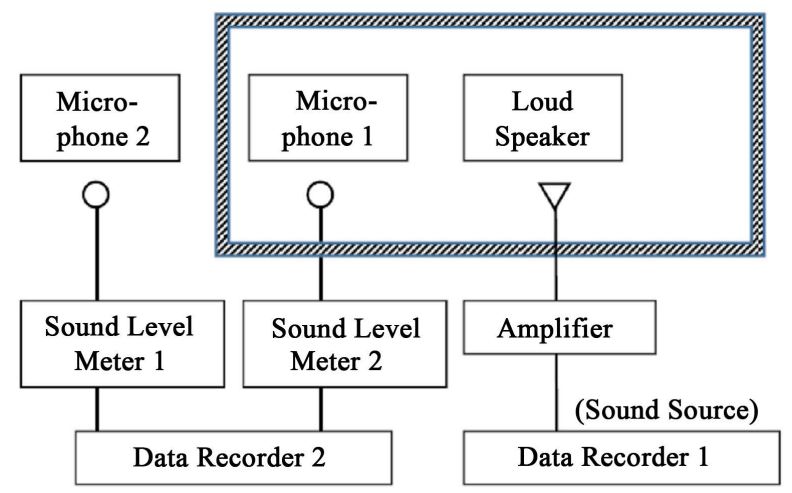

Figure 1. A schematic drawing of the experimental set up in sound insulation system.

After generating the music sound inside the house, the indoor and outdoor sound pressure levels were regarded as the input signal $x_{k}$ and the output observation $y_{k}$. The data were measured with a sampling interval of $1 \mathrm{~s}$. The statistics of the input signal and the output signal used in the experiment are shown in Table 1 and Table 2. Applying the proposed estimation method to actually observed output data quantized roughly with $1 \mathrm{~dB}, 2 \mathrm{~dB}$ and $3 \mathrm{~dB}$ widths as examples of fuzzy observation, the fluctuation wave form of the input signal was estimated. Comparison of the accuracy for the estimated probability distribution between the case considering a finite fluctuation range of the observation data and the case without consideration of the fluctuation range was discussed in our previous study [5].

Figures 2-4 show the estimation results of the fluctuation wave form of the input signal for Data 1 in a typical case of widely fluctuating signals, by applying the proposed algorithm to the quantized observation data with $1 \mathrm{~dB}, 2 \mathrm{~dB}$ and 3 $\mathrm{dB}$ widths. Furthermore, Figures 5-7 show the estimation results for Data 2 in a typical case of narrowly fluctuating signals. In these estimations, the finite number of expansion coefficients $A_{r s}(r, s \leq 2)$ are used for the simplification of the estimation algorithm. In these figures, the horizontal axis shows the discrete time $k$, of the estimation process, and the vertical axis expresses the sound pressure level. For comparison, the estimation results calculated by using the usual method are also shown in these figures. Since Kalman's filtering theory is widely used in the field of stochastic system, the extended Kalman filter [8] is also applied to the observation data as a trail by introducing the following observation model.

$$
z_{k}=a_{k} x_{k}+b_{k}+\varepsilon_{k}
$$

where $\varepsilon_{k}$ denotes the quantized noise. A uniform distribution within $[-q / 2, q / 2]$ ( $q$ : the quantized width) is assumed as the probability distribution of $\varepsilon_{k}$. Since the parameters $a_{k}$ and $b_{k}$ are unknown, these parameters are also estimated simultaneously with the input signal $x_{k}$ by introducing the following time transition models in addition to Equation (9):

$$
a_{k+1}=a_{k}, b_{k+1}=b_{k}
$$

The results estimated by the proposed method show good agreement with the 


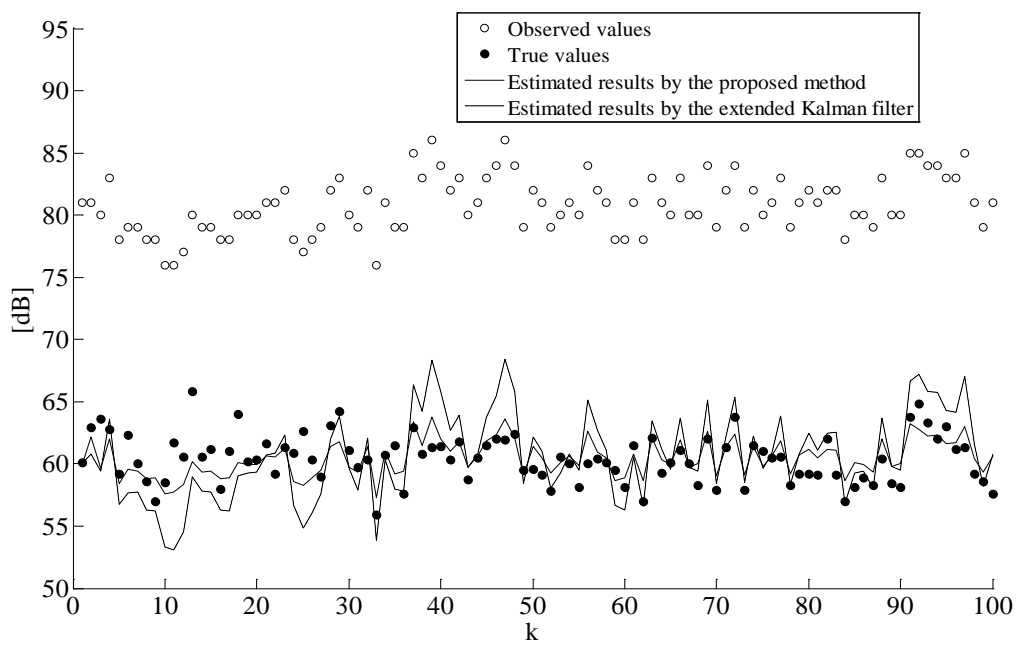

Figure 2. Estimation results for Data 1 of the input signal by applying the proposed method based on the quantized output observation data with $1 \mathrm{~dB}$ width.

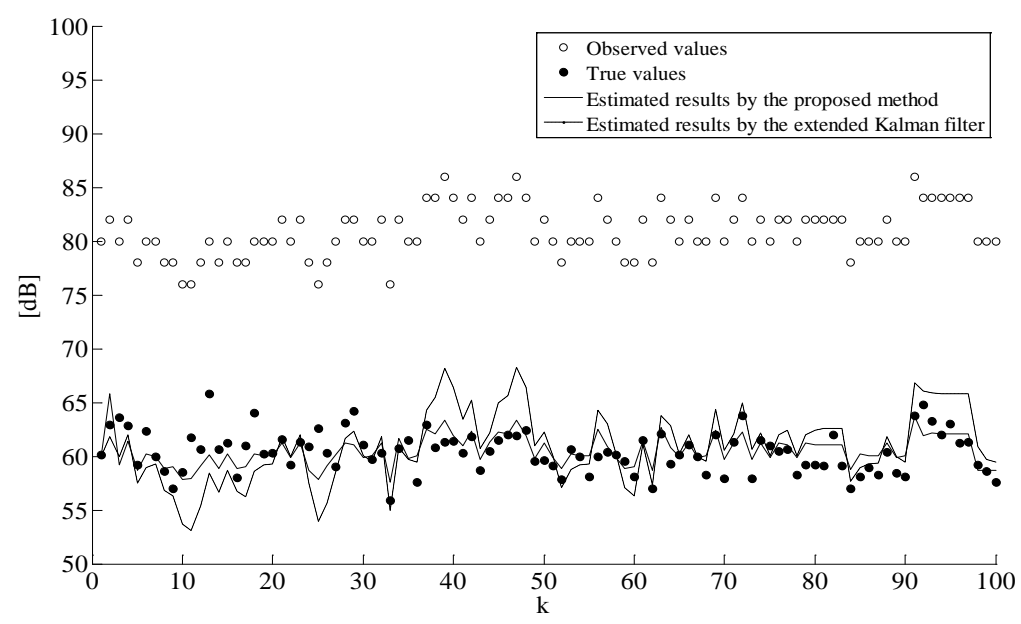

Figure 3. Estimation results for Data 1 of the input signal by applying the proposed method based on the quantized output observation data with $2 \mathrm{~dB}$ width.

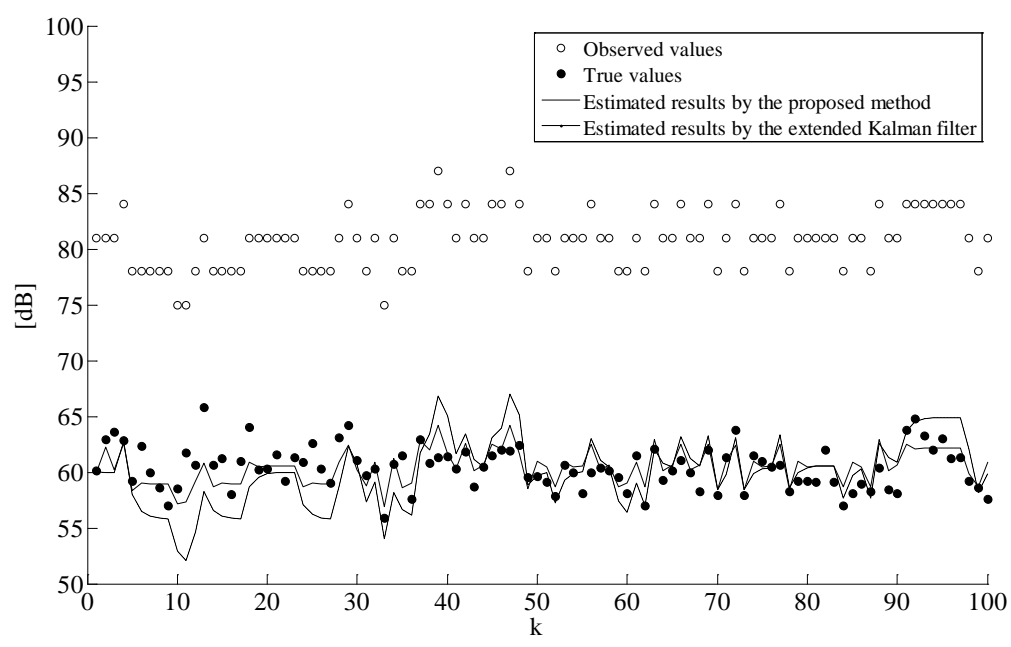

Figure 4. Estimation results for Data 1 of the input signal by applying the proposed method based on the quantized output observation data with $3 \mathrm{~dB}$ width. 


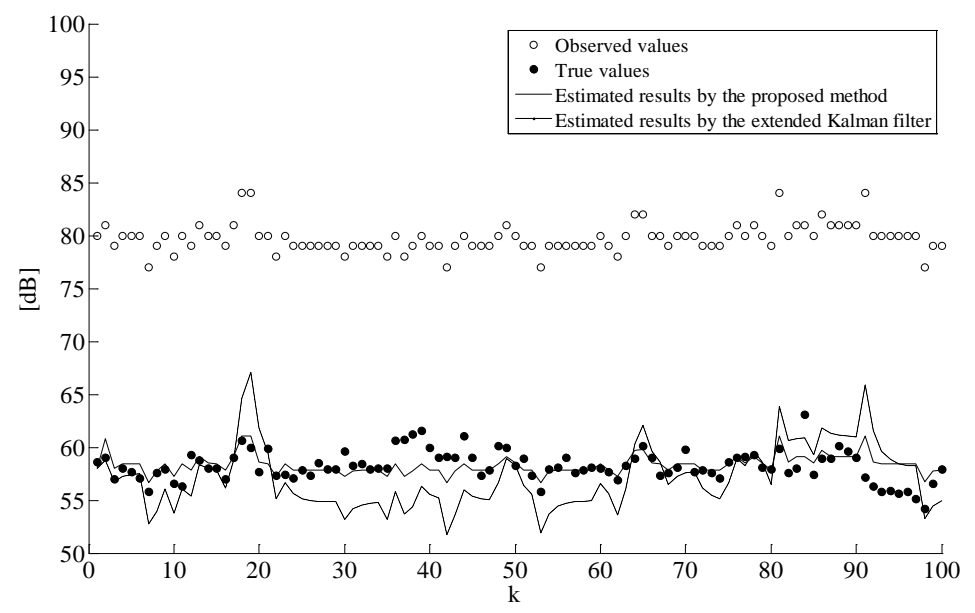

Figure 5. Estimation results for Data 2 of the input signal by applying the proposed method based on the quantized output observation data with $1 \mathrm{~dB}$ width.

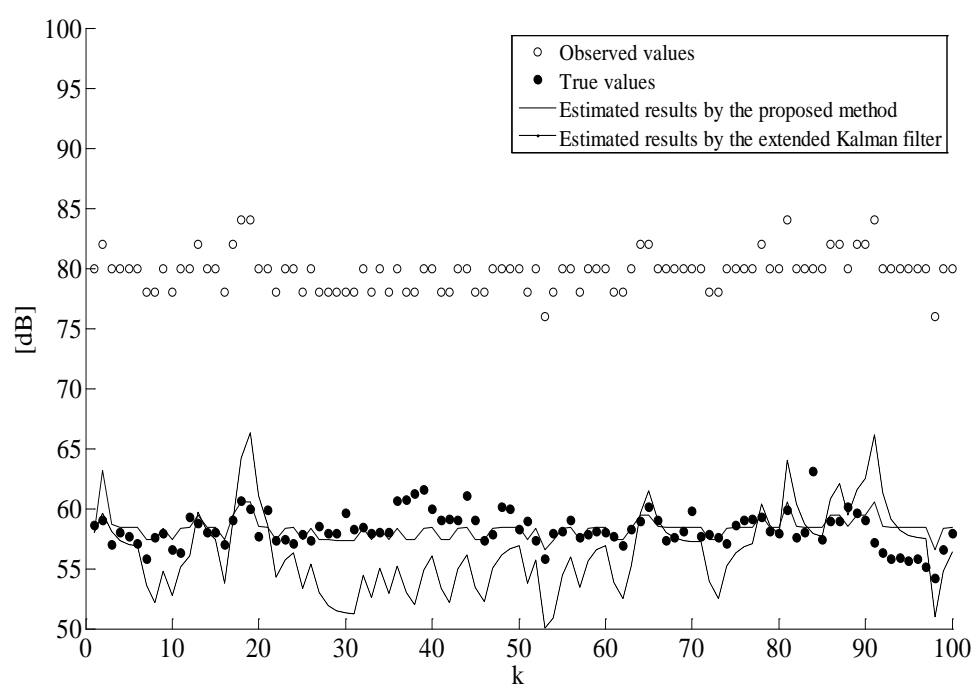

Figure 6. Estimation results for Data 2 of the input signal by applying the proposed method based on the quantized output observation data with $2 \mathrm{~dB}$ width.

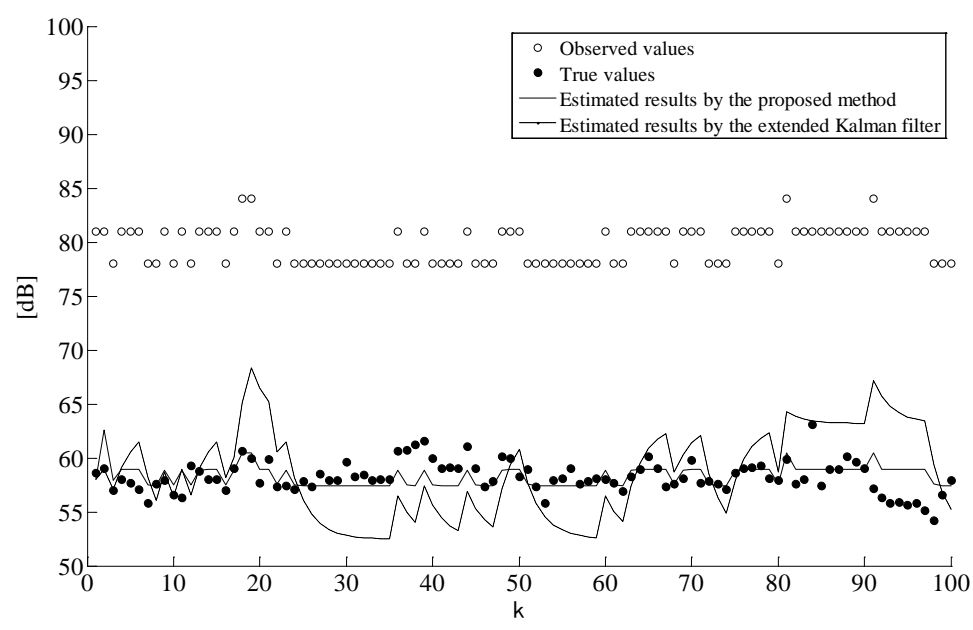

Figure 7. Estimation results for Data 2 of the input signal by applying the proposed method based on the quantized output observation data with $3 \mathrm{~dB}$ width. 
Table 1. Mean and standard deviation of the input signal (in $\mathrm{dB}$ ).

\begin{tabular}{cccccc}
\hline Data Number & Data 1 & Data 2 & Data 3 & Data 4 & Data 5 \\
\hline Mean Value & 80.8 & 79.6 & 80.4 & 82.4 & 80.8 \\
Standard Deviation & 2.25 & 1.31 & 2.38 & 2.26 & 2.50 \\
\hline
\end{tabular}

Table 2. Mean and standard deviation of the output signal (in dB).

\begin{tabular}{cccccc}
\hline Data Number & Data 1 & Data 2 & Data 3 & Data 4 & Data 5 \\
\hline Mean Value & 60.4 & 58.2 & 59.7 & 60.8 & 60.0 \\
Standard Deviation & 1.92 & 1.45 & 1.74 & 1.85 & 1.75 \\
\hline
\end{tabular}

true values. On the other hand, there are great discrepancies between the estimates based on the standard type dynamical estimation method (i.e., extended Kalman filter). For these differences on estimated results, the following reasons can be considered: 1) The standard method assumes the simple observation model in Equation (42). On the other hand, the proposed method introduces the conditional probability distribution in Equation (5) as the observation model, which can be considered the whole fluctuation of input and output signals; 2) The standard method is not considered the finite amplitude fluctuation of the observation data; 3) To consider the quantized observation by introducing fuzzy theory is more useful than the standard method by introducing the quantized noise.

The squared sums of the estimation error are shown in Tables 3-5. It can be found numerically that the proposed method is more useful than the extended Kalman filter.

\section{Conclusions}

In this study, based on the observed data with fuzziness and the finite level range, a new adaptive method for estimating the input signal for sound environment systems with unknown structure has been proposed. The proposed estimation method has been realized by introducing a system model of conditional probability type and the probability measure of fuzzy events. The proposed method has been applied to the estimation for the input signal of an actual sound environment system, and it has been experimentally verified that better results are obtained as compared with the standard estimation method without considering fuzzy theory.

The proposed approach is quite different from the traditional standard techniques. However, we are still in an early stage of development, and a number of practical problems are yet to be investigated in the future. These include: 1) application to a diverse range of sound signals in actual noise environment; 2) extension to cases with multi-noise sources, and 3) finding an optimal number of expansion terms for the expansion-based probability expressions adopted. 
Table 3. Comparison between the proposed method and the extended Kalman filter for root-mean squared error of the estimation based on the quantized observation data with $1 \mathrm{~dB}$ width (in $\mathrm{dB}$ ).

\begin{tabular}{cccccc}
\hline Data Number & Data 1 & Data 2 & Data 3 & Data 4 & Data 5 \\
\hline Proposed Methods & 1.61 & 1.39 & 1.32 & 1.25 & 1.45 \\
Extended Kalman Filter & 2.93 & 3.18 & 2.01 & 1.55 & 2.60 \\
\hline
\end{tabular}

Table 4. Comparison between the proposed method and the extended Kalman filter for root-mean squared error of the estimation based on the quantized observation data with $2 \mathrm{~dB}$ width (in $\mathrm{dB}$ ).

\begin{tabular}{cccccc}
\hline Data Number & Data 1 & Data 2 & Data 3 & Data 4 & Data 5 \\
\hline Proposed Methods & 1.63 & 1.47 & 1.34 & 1.38 & 1.50 \\
Extended Kalman Filter & 2.93 & 3.80 & 2.01 & 1.40 & 2.58 \\
\hline
\end{tabular}

Table 5. Comparison between the proposed method and the extended Kalman filter for root-mean squared error of the estimation based on the quantized observation data with $3 \mathrm{~dB}$ width (in $\mathrm{dB}$ ).

\begin{tabular}{cccccc}
\hline Data Number & Data 1 & Data 2 & Data 3 & Data 4 & Data 5 \\
\hline Proposed Methods & 1.63 & 1.53 & 1.39 & 1.53 & 1.51 \\
Extended Kalman Filter & 2.83 & 4.38 & 2.09 & 1.78 & 2.80 \\
\hline
\end{tabular}

\section{Acknowledgements}

The authors are grateful to Emeritus Prof. Mitsuo Ohta of Hiroshima University for his advice during this study. This work was supported in part by fund from the Grant-in-Aid for Scientific Research No.15K06116 from the Ministry of Education, Culture, Sports, Science and Technology-Japan.

\section{References}

[1] Ikuta, A., Masuike, H. and Ohta, M. (2005) A Digital Filter for Stochastic Systems with Unknown Structure and Its Application to Psychological Evaluation of Sound Environment. IEICE Transactions on Information and Systems, E88-D, 1519-1525. https://doi.org/10.1093/ietisy/e88-d.7.1519

[2] Ikuta, A. and Orimoto, H. (2014) Static and Dynamic Methods for Fuzzy Signal Processing of Sound and Electromagnetic Environment Based on Fuzzy Observations. In: Merelo, J.J., et al., Eds., Computational Intelligence, Studies in Computational Intelligence, Vol. 620, Springer, Berlin, 171-187.

[3] Ikuta, A. and Orimoto, H. (2008) Adaptive Method for State Estimation of Sound Environment System with Fuzzy Observation. Proceedings of 7 th IEEE International Conference on Cybernetic Intelligent Systems, London, 9-10 September 2008.

[4] Ikuta, A. and Orimoto, H. (2014) Stochastic Approach for Noise Suppression of Speech Signal by Considering Finite Range of Amplitude Fluctuation in Real Environment. International Journal of Applied Pattern Recognition, 1, 361-376. https://doi.org/10.1504/IJAPR.2014.068338

[5] Ikuta, A., Ohta, M. and Uzu, M. (2007) An Evaluation Method of Sound Environment Matched to the Fluctuation with a Finite Level Range in Our Actual Life and 
Its Application to Traffic Noise at Main Line. Transactions of the Society of Instrument and Control Engineers, 43, 529-535.

https://doi.org/10.9746/ve.sicetr1965.43.529

[6] Kalman, R.E. (1960) A New Approach to Linear Filtering and Prediction Problems. Journal of Basic Engineering, 82, 35-45. https://doi.org/10.1115/1.3662552

[7] Kalman, R.E. and Buch, R.S. (1961) New Results in Linear Filtering and Prediction Theory. Journal of Basic Engineering, 83, 95-108. https://doi.org/10.1115/1.3658902

[8] Kushner, H.J. (1967) Approximations to Optimal Nonlinear Filters. IEEE Transactions on Automatic Control, 12, 546-556. https://doi.org/10.1109/TAC.1967.1098671

[9] Bell, B.M. and Cathey, F.W. (1993) The Iterated Kalman Filter Update as a Gaussian-Newton Methods. IEEE Transactions on Automatic Control, 38, 294-297. https://doi.org/10.1109/9.250476

[10] Julier, S. and Uhlmann, J. (2004) Unscented Filtering and Nonlinear Estimation. Proceedings of IEEE, 92, 401-422. https://doi.org/10.1109/JPROC.2003.823141

[11] Kitagawa, G. (1996) Montecarlo Filter and Smoother for Non-Gaussian Nonlinear State Space Models. Journal of Computational and Graphical Statistics, 5, 1-25.

[12] Ikuta, A., Tokhi, M.O. and Ohta, M. (2001) A Cancellation Method of Background Noise for a Sound Environment System with Unknown Structure. IEICE Transactions on Fundamentals of Electronics, Communications and Computer Sciences, E84-A, 457-466.

[13] Ikuta, A., Orimoto, H., Siddique, N.H. and Maguire, L.P. (2015) Statistical Evaluation of Complex Sound Environment with Background Noise. International Journal of Acoustics and Vibration, 20, 41-46. https://doi.org/10.20855/ijav.2015.20.1367

[14] Guoshen, Y. (2012) Solving Inverse Problems with Piecewise Linear Estimators: From Gaussian Mixture Models to Structured Sparsity. IEEE Transactions on Image Processing, 21, 2481-2499. https://doi.org/10.1109/TIP.2011.2176743

[15] Zadeh, L.A. (1968) Probability Measures of Fuzzy Events. Journal of Mathematical Analysis and Applications, 23, 421-427. https://doi.org/10.1016/0022-247X(68)90078-4

[16] Box, G.E.P., Jenkins, G.M., Reinsel, G.C. and Ljung, G.M. (2016) Time Series Analysis-Forecasting and Control. 5th Edition, John Wiley \& Sons, New York.

[17] Krysicki, W. (1999) On Some New Properties of the Beta Distribution. Statistics \& Probability Letters, 42, 131-137. https://doi.org/10.1016/S0167-7152(98)00197-7

[18] Ohta, M. and Koizumi, T. (1968) General Statistical Treatment of Response of a Non-Linear Rectifying Device to a Stationary Random Input. IEEE Transactions on Information Theory, 14, 595-598. 
Submit or recommend next manuscript to SCIRP and we will provide best service for you:

Accepting pre-submission inquiries through Email, Facebook, LinkedIn, Twitter, etc. A wide selection of journals (inclusive of 9 subjects, more than 200 journals)

Providing 24-hour high-quality service

User-friendly online submission system

Fair and swift peer-review system

Efficient typesetting and proofreading procedure

Display of the result of downloads and visits, as well as the number of cited articles Maximum dissemination of your research work

Submit your manuscript at: http://papersubmission.scirp.org/

Or contact jsea@scirp.org 